\title{
HCCI DE ETANOL HIDRATADO EM UM CILINDRO DEDICADO DE UM MOTOR DIESEL USANDO RECIRCULAÇÃO DOS GASES DA EXAUSTÃO
}

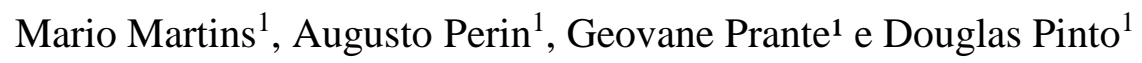 \\ ${ }^{1}$ Universidade Federal de Santa Maria \\ E-mails: mario@mecanica.ufsm.br, augusto.t.perin@gmail.com.br, \\ geovanefrizzo@gmail.com e douglasubertpinto@hotmail.com
}

\section{RESUMO}

Etanol de baixo custo com elevados percentuais de água é um combustível que pode ser facilmente produzido e que possui um grande potencial para substituir combustíveis fósseis, desta forma, minimizando as emissões de efeito estufa. Entretanto, ele apresenta diversos desafios de ignição no que diz respeito ao percentual de água, temperatura ambiente, razão de compressão e outros parâmetros específicos do motor. Conceitos avançados de combustão como ignição de carga homogênea por compressão (HCCI) mostram ser muito tolerantes à adição de água no combustível devido a sua natureza de não propagação de chama. Além disso, a rápida e de baixa temperatura combustão em HCCI reduz as perdas de calor para as paredes do cilindro, dessa forma aumentando eficiência térmica enquanto reduzindo a formação de óxidos de nitrogênio (NOx). Nesse sentido, o presente trabalho demonstra a operação de um motor gerador de energia elétrica de 3 cilindros em que dois cilindros operam no ciclo diesel convencional (CDC) e fornecem gases do escape reciclados (EGR) para o último cilindro operando com etanol hidratado e combustão HCCI. Condições estáveis de operação foram alcançadas de 0 a 4 bar IMEP com diferentes combinações de razão ar/combustível, taxa de EGR e diferentes percentuais de água no etanol. Valores de eficiência excedendo os de motores diesel foram alcançados.

\section{INTRODUÇÃO}

É um claro consentimento de que a atividade humana está aquecendo o planeta. $95 \%$ da comunidade científica concorda que, desde a década de 1950, a temperatura média subiu a taxas sem precedentes. De acordo com o relatório do Painel Intergovernamental de Mudanças Climáticas de 2007 (IPCC), esse aquecimento observado se deve principalmente aos gases de efeito estufa, como aqueles expelidos de motores de combustão interna, por exemplo, dióxido de carbono (CO2). A queima de combustíveis fósseis representou um aumento de $70 \%$ nos gases de efeito estufa nas últimas quatro décadas. [1]

A inexistência de uma legislação nacional sobre as emissões de motores geradores de energia, em sua grande maioria motores a óleo diesel, permite a produção de altos níveis de gases poluentes, causando assim um grande impacto ambiental. Nesse cenário, motores a ignição por compressão de carga homogênea (HCCI) podem preencher a falta de motores menos poluentes nas unidades geradoras de energia. Os motores HCCI combinam as características dos motores de ignição por centelha (SI) e de ignição por compressão (CI), aproveitando 
ambos os modos de combustão, fornecendo um motor de alta eficiência como o diesel e de baixos níveis de emissão, principalmente de óxidos de nitrogênio (NOx). [2]

De acordo com [3], [4], os motores HCCI são tolerantes a uma grande variedade de combustíveis, apresentando resultados satisfatórios com vários álcoois e combustíveis fósseis, como gasolina e diesel. No entanto, o etanol com altos níveis de hidratação é um combustível renovável, facilmente produzido e de baixo custo que oferece várias vantagens e pode substituir os combustíveis fósseis comuns. Durante a produção do etanol, uma grande quantia de energia é gasta na destilação e desidratação, tornando-se exponencial o aumento dos custos nos últimos $20 \%$ de água. [5] Além disso, levando-se em conta o processo geral de produção de etanol a partir de biomassa, o balanço de carbono, "well-to-wheel", é extremamente vantajoso, justificando assim o uso do etanol hidratado como combustível. [6]

Diferentemente dos motores SI, onde o processo de combustão depende da propagação da chama e dos motores CI que dependem da combustão por difusão, os motores HCCI têm o combustível e o ar misturados antes de entrar no cilindro e à medida que o pistão sobe, a temperatura aumenta, atingindo a temperatura de autoignição do combustível. A ignição inicia-se em múltiplos pontos na câmara de combustão, ocorrendo assim a combustão da carga homogênea. [7]

Este processo de combustão pode ser consideravelmente rápido devido ao fato da carga entrar em combustão de uma só vez, resultando em taxas extremamente altas de aumento de pressão e liberação de calor. Esses fatores beneficiam a eficiência térmica geral, uma vez que as altas taxas de aumento de pressão (PRR) encurtam a duração da combustão, se aproximando de uma combustão a volume constante, diminuindo as perdas de calor para as paredes do cilindro e sistema de arrefecimento, aumentando assim o trabalho líquido realizado. [8] No entanto, esses altos valores de PRR podem resultar em excessivo ruído de combustão e, portanto, entrar em detonação. Neste sentido, os motores HCCI geralmente requerem alguma forma de diluição da carga para controlar este aumento de pressão.

Contudo, existem vários problemas de ignição relacionados à temperatura de admissão, razão de compressão e outros parâmetros específicos do motor, levando ao desenvolvimento de várias abordagens. [9] Um dos problemas mais difíceis de controlar é o evento de autoignição. Diferentemente dos motores SI, em que a ignição é controlada pelo instante da centelha, e dos motores CI, cuja ignição depende do instante de injeção de combustível, os motores HCCI são amplamente dependentes da cinética química e da composição e temperatura do cilindro. [10] [11]

Em estudos anteriores, [12], [13] os autores obtiveram resultados bem-sucedidos com o aprisionamento dos gases de exaustão com o uso de cruzamento negativo de válvula (NVO), aquecendo assim a pré-mistura homogênea dentro do cilindro e atingindo a temperatura de autoignição do combustível. Outras técnicas, como o uso de dispositivos de aquecimento externos para aquecer o ar de admissão, foram estudadas por [14]. Estudos anteriores [15] mostraram que outra estratégia comum é o uso da recirculação dos gases de escape (EGR) para reciclar o calor rejeitado na exaustão afim de inflamar a mistura dentro do cilindro. Ambos métodos realizam o aquecimento da mistura ar-combustível e a diluição da carga devido aos altos teores de gases inertes, como $\mathrm{CO} 2$, controlando assim a ocorrência de detonação, reduzindo a taxa de elevação da pressão. [16], [17] 
Diferentes estratégias e inúmeros trabalhos foram feitos com relação ao HCCI, no entanto, muito pouco foi feito para investigar a combustão HCCI de etanol hidratado, com EGR proveniente de combustão diesel para promover a autoignição do combustível em uma unidade geradora de energia. Este estudo propõe uma investigação do desempenho e eficiência de um motor a diesel original gerador de energia convertido para trabalhar com HCCI de etanol hidratado e EGR.

\section{METODOLOGIA}

O aparato experimental consistiu de um motor diesel MWM D229, três cilindros naturalmente aspirado de uma unidade de geração de energia. Um dos cilindros foi isolado dos demais para operar somente com etanol hidratado através de uma injeção de combustível no coletor (PFI). Os dois cilindros remanescentes se mantiveram operando com diesel com injeção direta de diesel (DI). Uma borboleta foi instalada para controlar a admissão de ar no cilindro operando com etanol hidratado e válvulas globo foram instaladas para controlar a quantidade de EGR dos cilindros diesel para o cilindro operando com etanol hidratado, como mostra o modelo representativo na Figura 1.

Uma injeção programável foi usada para controlar a injeção de etanol hidratado. Nenhuma alteração nas geometrias de pistão e câmara de combustão foi realizada, com exceção de espaçadores utilizados para variar a razão de compressão no cilindro dedicado a etanol hidratado para se avaliar duas razões de compressão diferentes. Os parâmetros originais e modificados são apresentados na Tabela 1 .

Tabela 1. Parâmetros do motor

\begin{tabular}{|c|c|c|}
\hline & Standard & Modified \\
\hline Cylinders & 3 and 2 & HCCI \\
\hline Ignition & CI & Wet Ethanol \\
\hline Fuel & Diesel & PFI \\
\hline Injection System & DI & Water-cooled \\
\hline Compression Ratio & $16.6: 1$ & 102 \\
\hline Cooling & \multicolumn{2}{|c|}{120} \\
\hline Bore (mm) & \multicolumn{2}{|c|}{207} \\
\hline Stroke (mm) & 2 \\
\hline Rod Length (mm) & \multicolumn{2}{|c|}{980.3} \\
\hline Valves per Cylinder & \multicolumn{2}{|c|}{ head } \\
\hline Swept Volume (cm $\left.{ }^{3}\right)$ & Centrally located omega-shaped piston bowl with flat cylinder \\
\hline Combustion Chamber & 1800 \\
\hline Engine Speed (rpm) & \multicolumn{2}{|c|}{} \\
\hline
\end{tabular}

Um software dedicado para leitura de sinais foi utilizado para a aquisição de dados. A operação com HCCI no cilindro operando com etanol hidratado foi limitada devido ao elevado ruído de combustão em altas cargas. Enquanto isso, em baixas cargas houve limitação por misfire, tendo máxima covariância (COV) do IMEP de aproximadamente $3 \%$. 


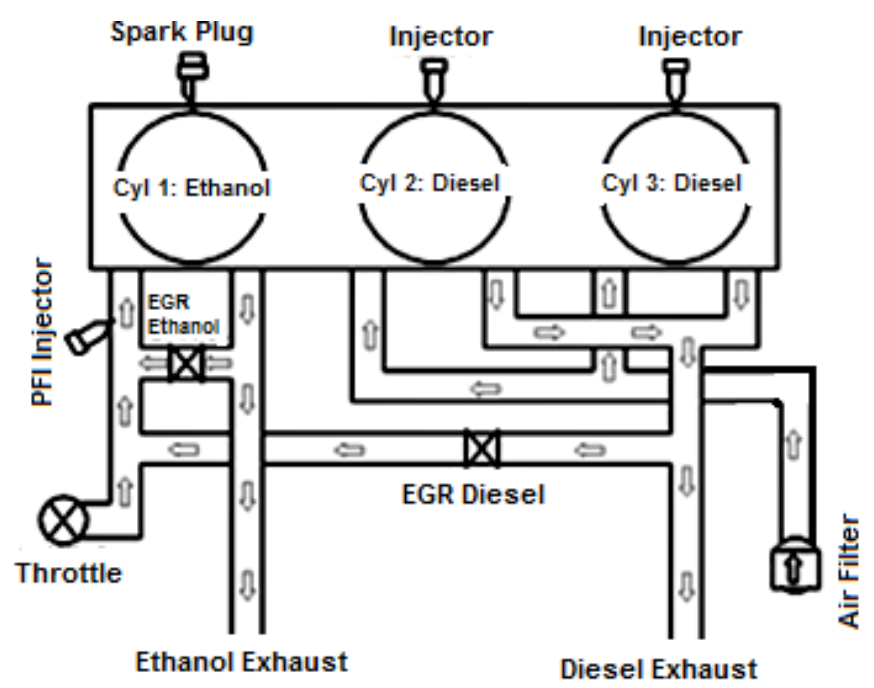

Figura 1. Esquema representativo do aparato experimental

EGR foi empregada para diluir a mistura ar-combustível dentro do cilindro e, assim, diminuir a taxa de elevação de pressão em combustão HCCI.[18] Dentre os vários métodos para se estimar a fração de EGR, o escolhido nesse trabalho é baseado na análise de espécies representativas como $\mathrm{CO} 2$ através da amostragem alternada de gases de admissão e exaustão.[19]-[21] O sistema consiste em duas válvulas solenoides que permitem uma fração da amostra ser medida em um analisador de gases, cujo princípio de funcionamento é espectroscopia de infravermelhos através da transformada de Fourier (FTIR).

A pressão de cilindro foi adquirida com um sensor de pressão piezoelétrico, com alta taxa de aquisição, enquanto pressões instantâneas de admissão e escape foram medidas com sensores piezoresistivos. Um encoder incremental com 3600 pulsos por revolução foi utilizado. Uma placa de aquisição de dados foi usada para a aquisição dos dados de pressão e temperatura, bem como monitoramento em tempo real.

Consumo de combustível foi medido usando uma bureta graduada e a razão ar-combustível foi gravada usando um sensor de oxigênio de banda larga. Frações de água no etanol de $10 \%$ a $30 \%$ foram testadas em uma velocidade constante de $1800 \mathrm{rpm}$. Todos os dados de combustão apresentados são médias feitas de 100 ciclos consecutivos do motor.

\section{RESULTADOS}

A pressão média efetiva indicada (IMEP) para ambas razões de compressão foram avaliadas variando a carga de 0 a $400 \mathrm{kPa}$ no cilindro dedicado a etanol hidratado. Esses valores foram medidos através de três percentuais de hidratação do etanol, sendo $10 \%, 20 \%$ e $30 \%$ de volume de água em etanol. Maiores cargas em combustão HCCI não foram alcançadas devido às altas taxas de elevação de pressão (PRR) e ao alto ruído de combustão, mesmo tendo uma mistura altamente diluída e altamente pobre (lambda > 1). Esses resultados foram encontrados para todas as misturas de etanol e água testadas, independentemente do percentual de água, e todas as condições foram limitadas por detonação.

Maiores valores de IMEP poderiam ser alcançados utilizando trocadores de calor para a recirculação dos gases do escape (EGR). Dessa forma, controlando a combustão e tornando-a mais estável em condições de alta carga. Figura 2 e 3 mostram que a razão de compressão 
mais elevada de 16.6:1 reduziu a temperatura necessária de admissão para todas as misturas e cargas avaliadas. Essa tendência é razoável uma vez que a mistura se aquece durante a compressão com um menor volume morto.

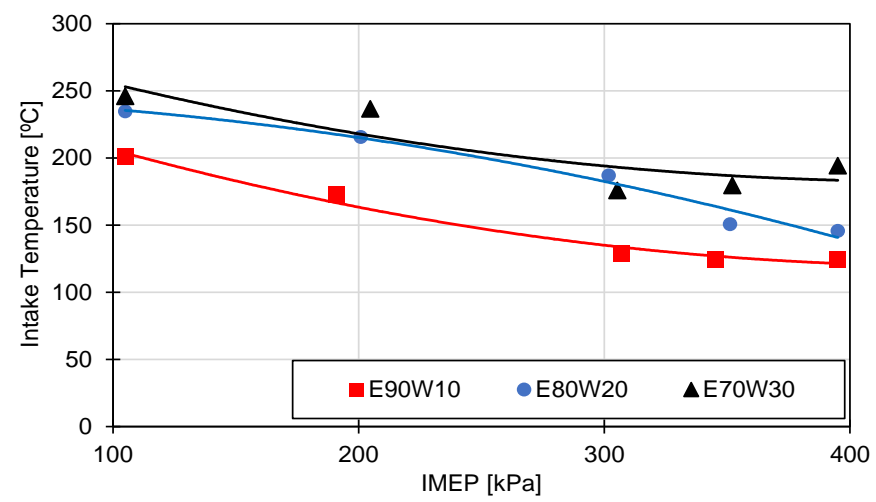

Figura 2. Temperatura de admissão em função da carga para razão de compressão de 14:1

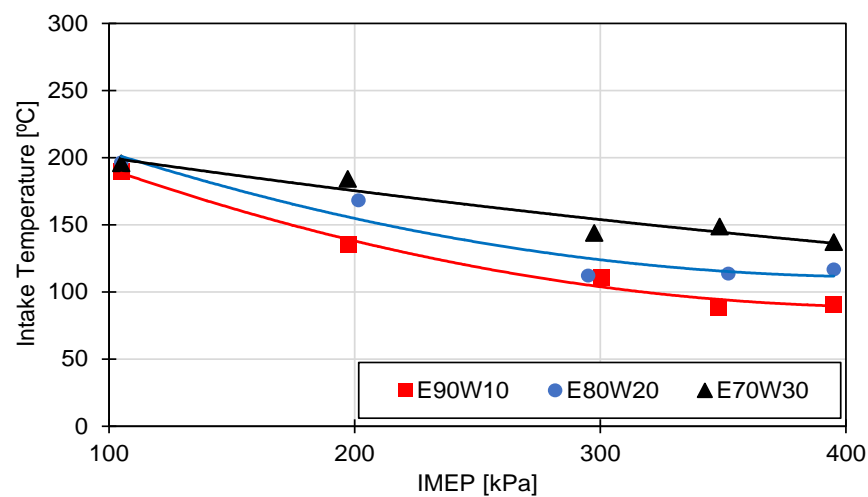

Figura 3. Temperatura de admissão em função da carga para razão de compressão de 16.6:1

Considerando a água no etanol, quanto maior o percentual, maior o calor necessário para promover a autoignição do combustível e assim, mais EGR foi demandada dos cilindros operando em combustão diesel. Isso pode ser descrito pelo alto calor de vaporização da água. O percentual de EGR é mostrado nas Figuras 4 e 5. Há uma clara tendência entre o percentual de hidratação e a quantidade de EGR necessária e a mesma pode ser vista para ambas razões de compressão. Por outro lado, o aumento na carga reduziu a quantidade de calor necessário e, consequentemente, a demanda de EGR.

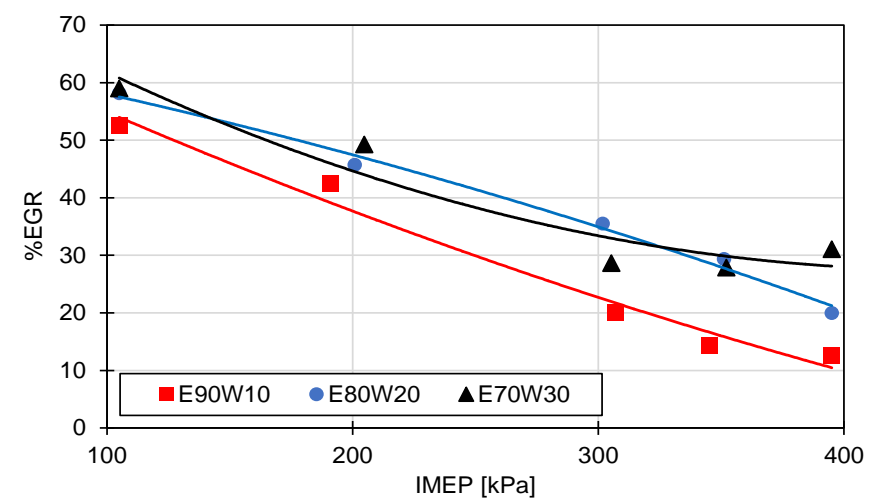

Figura 4. EGR em função da carga para razão de compressão de 14:1 


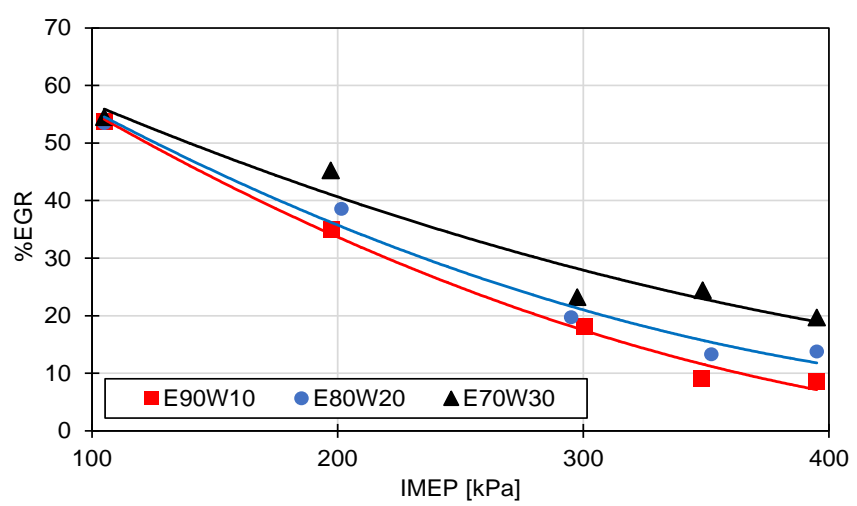

Figura 5. EGR em função da carga para razão de compressão de 16.6:1

A respeito da eficiência indicada, o aumento do percentual de água promoveu um aumento de eficiência até uma adição de $20 \%$ de água no etanol. Uma vez que a água absorve calor da mistura ar-combustível dentro do cilindro, a fase da combustão foi adiada e o ângulo do virabrequim de $50 \%$ de fração de massa queimada (CA50) foi atrasado, beneficiando assim a eficiência indicada. Entretanto, com uma adição de 30\% de água em etanol, houve um decréscimo na eficiência indicada. Isso pode ser atribuído à uma combustão instável, pouco eficiente, devido aos altos níveis de hidrocarbonetos não queimados (uHC).

Comparando as duas razões de compressão, os resultados de eficiência indicada mostrados na Figura 6 foram menores para todas as misturas de etanol e água na razão de compressão mais elevada de 16.6:1. Não somente, o consumo específico de combustível indicado (ISFC) foi também mais elevado para a mesma razão de compressão, como mostra a Figura 7. Quanto maior foi a adição de água, maior o ISFC.

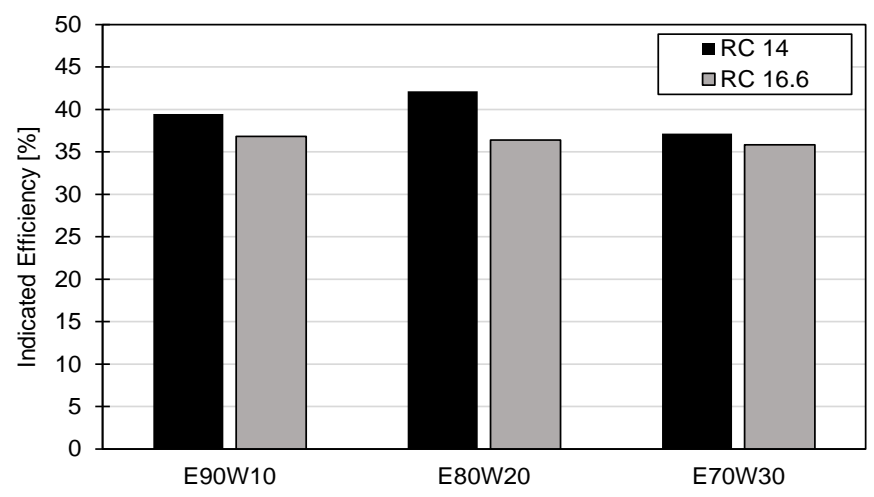

Figura 6. Eficiência indicada para ambas razões de compressão

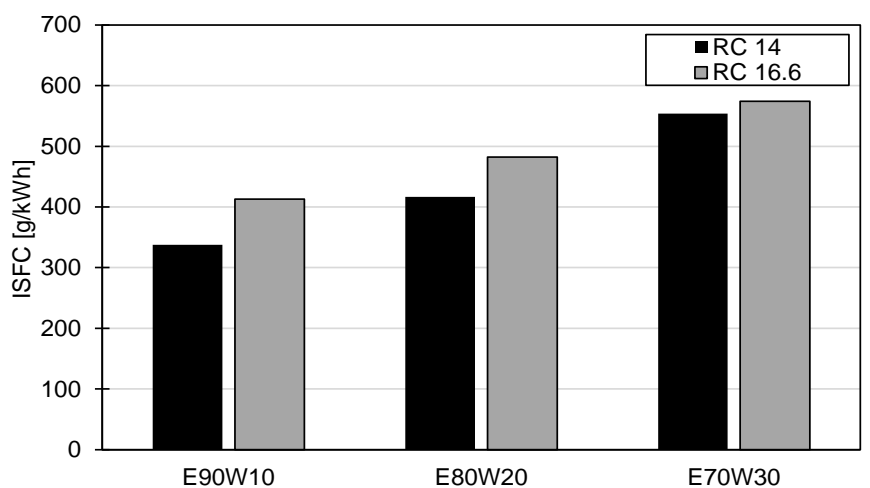

Figura 7. ISFC para ambas razões de compressão 
As Figuras 8 e 9 ilustram o CA50 para ambas razões de compressão. Exceto as condições de alta carga com baixas frações de água no etanol, a grande maioria dos pontos de operação para a razão de operação de 16.6:1 tiveram seu ponto de CA50 antes do ponto morto superior (TDC), dessa forma aumentando o trabalho de compressão e assim diminuindo a eficiência indicada. Além disso, é possível visualizar um deslocamento nas curvas de CA50 para as duas razões de compressão analisadas, mostrando que a menor razão de compressão pode realizar maior trabalho útil de expansão para a mesma condição de carga.

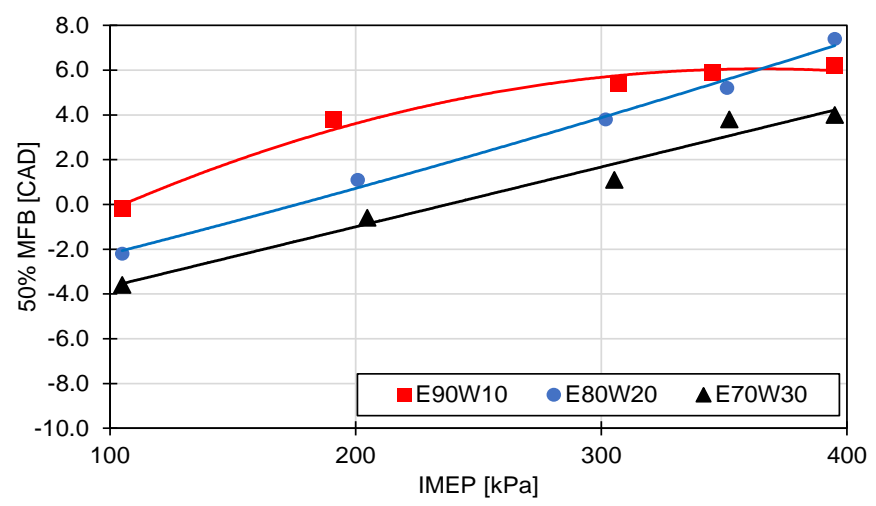

Figura 8. CA50 em função da carga para razão de compressão de 14:1

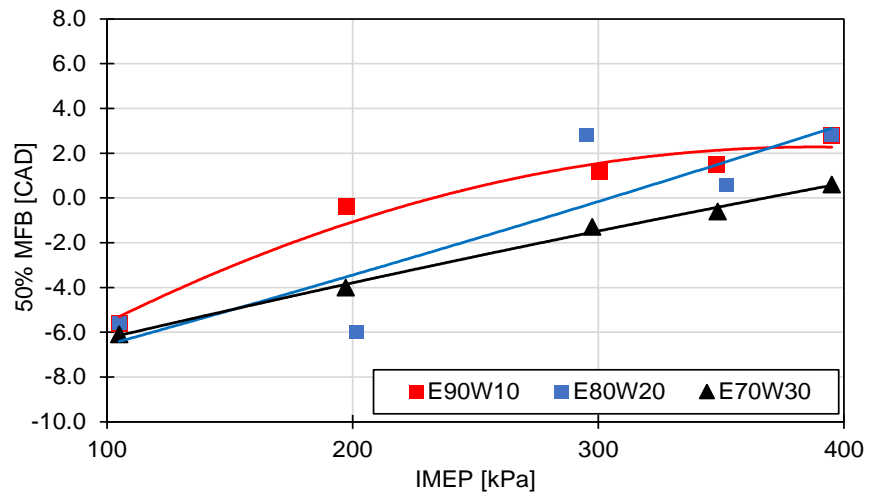

Figura 9. CA50 em função da carga para razão de compressão de 16.6:1

Figura 10 e 11 expõem o início da combustão (SOI), calculado a partir do ângulo de virabrequim de $2 \%$ de fração de massa queimada (CA2). De modo geral, a combustão começou perto do TDC e em condições de baixa carga teve seu começo ainda mais adiantado. Para a razão de compressão mais elevada, o SOI teve seu começo posteriormente, principalmente devido à mais altas temperaturas alcançadas dentro do cilindro resultante da compressão. Figuras 12 e 13 ilustram a duração de combustão, que nesse caso foram calculadas através do ângulo de virabrequim entre $10 \%$ e $90 \%$ da fração de massa queimada. $\mathrm{Na}$ maior parte das condições, a duração de combustão permaneceu abaixo de $8^{\circ}$ de virabrequim, concordando consistentemente com estudos previamente realizados sobre combustão HCCI.[22] Entretanto, alguns casos em carga parcial para a razão de compressão de 14:1 se mantiveram perto de $10^{\circ}$ de virabrequim. Pode-se inferir que a maior duração de combustão foi devido a uma mistura mais diluída e mais pobre nessas condições de operação. 


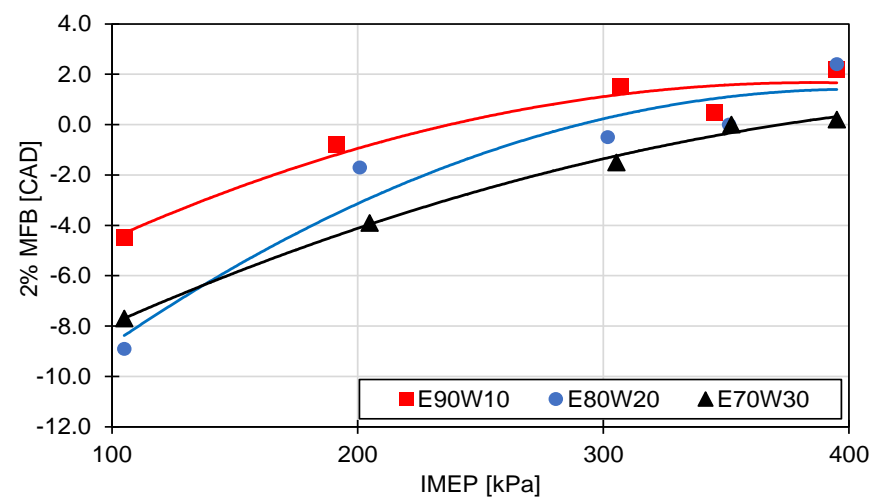

Figura 10. SOI em função da carga para razão de compressão de 14:1

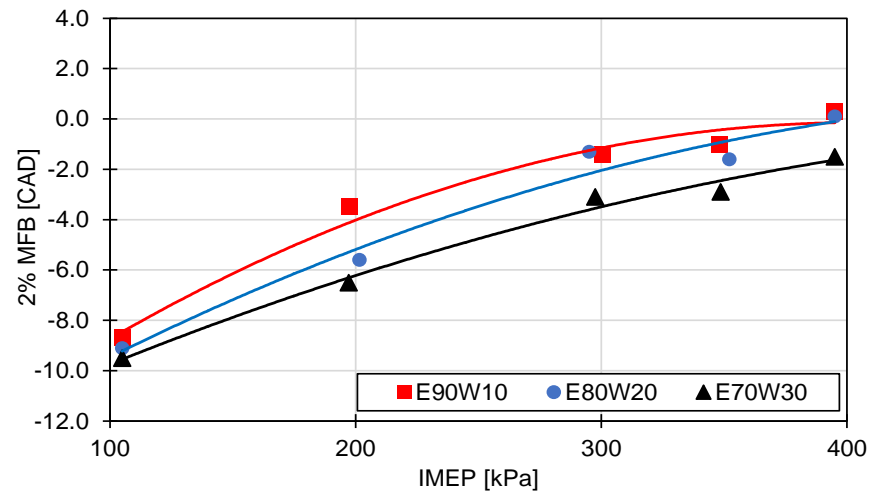

Figura 11. SOI em função da carga para razão de compressão de 16.6:1

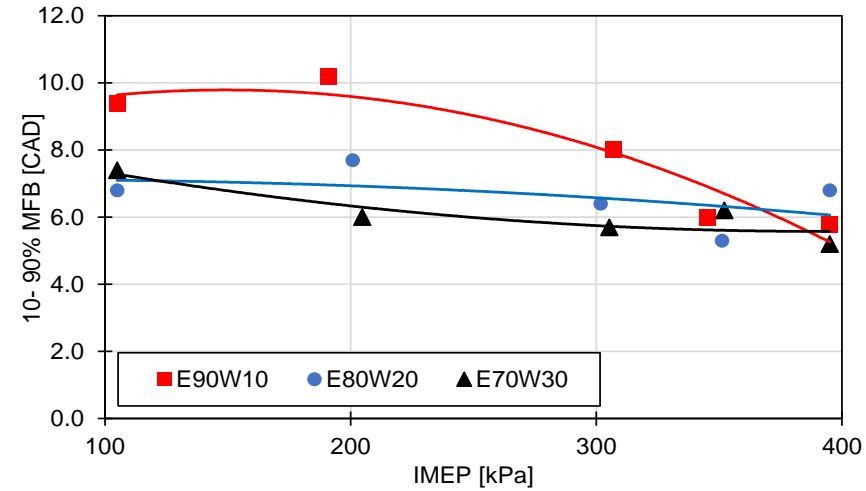

Figura 12. Duração de combustão em função da carga para razão de compressão de 14:1

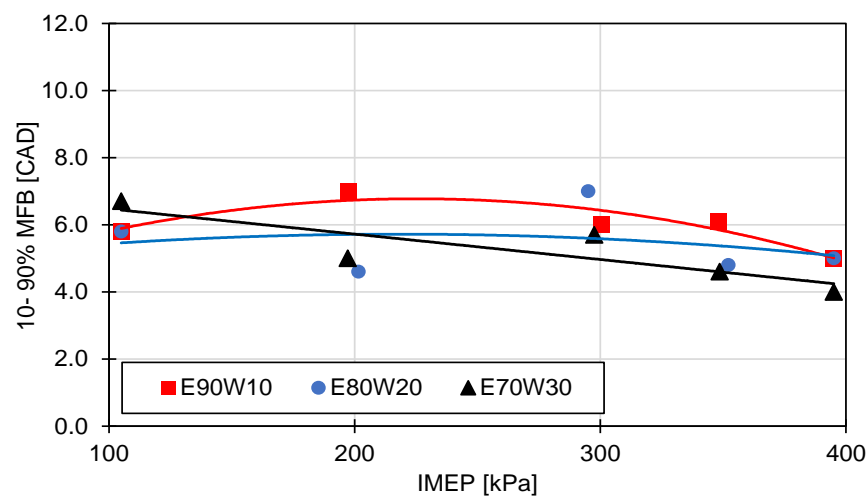

Figura 13. Duração de combustão em função da carga para razão de compressão de 16.6:1

A taxa de liberação de calor (HRR) para ambas razões de compressão estão plotadas nas Figuras 14 e 15. Ambas as curvas mostraram um rápido perfil de liberação de calor dado por uma rápida combustão, característico de uma combustão HCCI. No geral, a adição de água 
reduziu todos os valores de liberação de calor. Entretanto, com 30\% de água em etanol houve uma tendência anormal, apresentando valores de HRR maiores do que para os outros dois combustíveis. Pode ser dito que uma combinação de fatores como temperatura de admissão, composição do combustível e percentual de EGR dos cilindros a diesel podem influenciar a combustão HCCI. Neste caso, a maior temperatura de admissão pode ter tido um papel predominante nessa tendência.

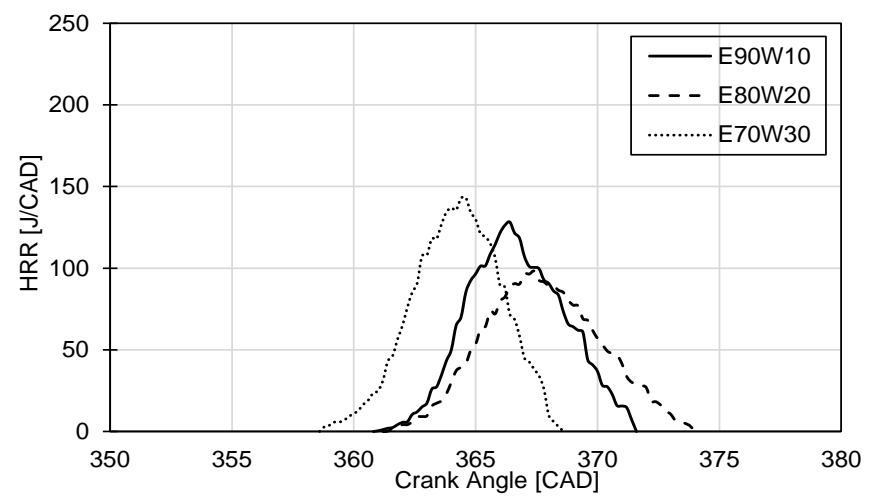

Figura 14. HRR em função do ângulo de virabrequim para razão de compressão de 14:1

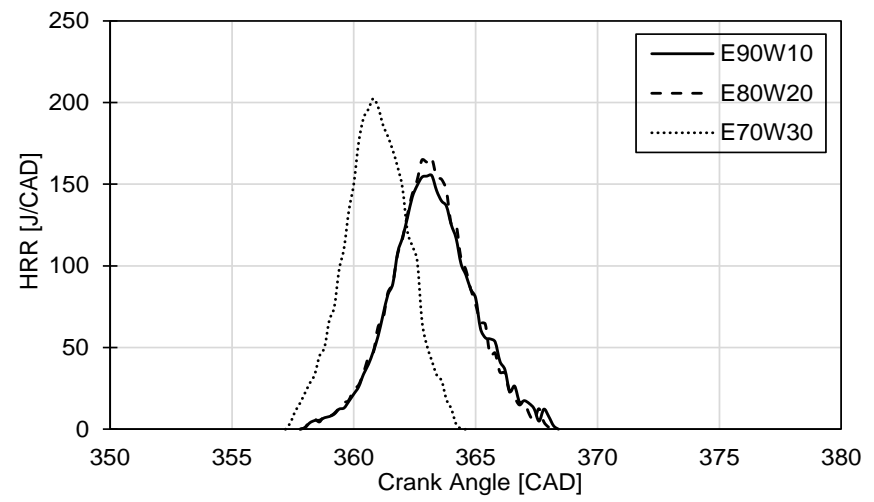

Figura 15. HRR em função do ângulo de virabrequim para razão de compressão de 16.6:1

\section{CONCLUSÃO}

Este trabalho discutiu o estudo experimental de um motor originalmente diesel operando com um cilindro com combustão HCCI e etanol hidratado com vários percentuais de hidratação. Calor proveniente da descarga dos cilindros diesel foi recuperado através da recirculação dos gases do escape para promover a autoignição da mistura ar-combustível no cilindro HCCI de um gerador de energia. Os resultados foram comparados entre as duas razões de compressão analisadas e parâmetros de performance e combustão foram avaliados.

No que diz respeito a performance, esse estudo mostrou que os maiores valores de eficiência indicada foram maiores para uma adição de $20 \%$ de água em etanol. Valores de até $42 \%$ de eficiência indicada foram alcançados para uma razão de compressão de 14:1, se aproximando ou até mesmo excedendo eficiências indicadas de motores diesel. No quesito combustão, a mesma não foi significativamente afetada pela adição de água, tendo combustão estável em quase todos os casos e apresentando durações de combustão entre $6^{\circ}$ e $8^{\circ}$ de virabrequim. 
Além dos benefícios que a combustão HCCI demonstrou no presente trabalho, várias limitações foram encontradas no que diz respeito a aplicação real em motores para geração de energia. Limitações de potência e elevado consumo volumétrico de combustível podem ser citados. Além disso, problemas relacionados a corrosão de partes mecânicas e contaminação de óleo lubrificante pela água devem ser considerados.

\section{REFERÊNCIAS}

[1] REAY, Dave et al. Climate change 2007: spring-time for sinks. Nature, Cambridge, v. 446, n. 7137, p. 727-8, 2007.

[2] BHAGATWALA, Ankit; CHEN, Jacqueline H.; LU, Tianfeng. Direct numerical simulations of HCCI/SACI with ethanol. Combustion and Flame, v. 161, n. 7, p. 1826-1841, 2014.

[3] XIE, Hui et al. Comparison of HCCI Combustion Respectively Fueled with Gasoline, Ethanol and Methanol through the Trapped Residual Gas Strategy. 2006.

[4] ZHENG, Ming et al. Investigation of butanol-fuelled HCCI combustion on a high efficiency diesel engine. Energy Conversion and Management, v. 98, p. 215-224, 2015.

[5] SAXENA, Samveg et al. Optimal operating conditions for wet ethanol in a HCCI engine using exhaust gas heat recovery. Applied Energy, v. 116, p. 269-277, 2014.

[6] MACK, J.Hunter et al. Experimental investigation of butanol isomer combustion in Homogeneous Charge Compression Ignition (HCCI) engines. Applied Energy, v.165

[7] MAURYA, Rakesh Kumar; AGARWAL, Avinash Kumar. Experimental study of combustion and emission characteristics of ethanol fuelled port injected homogeneous charge compression ignition (HCCI) combustion engine. Applied Energy, v. 88, n. 4, p. 1169-1180, 2011.

[8] GHAZIKHANI, M. et al. An Experimental Study on the Effect of EGR and Engine Speed on CO and HC Emissions of Dual Fuel HCCI Engine. v. 3, n. 4, p. 357-362, 2009.

[9] MARTINS, Mario E. S. et al. Conversion of a diesel engine to operate with HCCI of wet ethanol in one of the cylinders. ABMC Internation Congress of Mechanical Engineering, 2015.

[10] SHAHBAKHTI, Mahdi; KOCH, Charles Robert. Thermo-kinetic combustion modeling of an HCCI engine to analyze ignition timing for control applications. Spring Technical Meeting Combustion Institute/Canadian Section, [s. 1.], p. 1-7, 2007.

[11] TURÁNYI, Tamás; TOMLIN, Alison S. Analysis of Kinetic Reaction Mechanisms.

[12] HE, Bang-quan et al. An Experimental Study on HCCI Combustion in a FourStroke Gasoline Engine with Reduced Valve Lift Operations. 2005

[13] MARTINS, Mario. Investigation of Performance and Characteristics of a MultiCylinder Gasoline Engine with Controlled Auto-Ignition Combustion in Naturally Aspirated and Boosted Operation, 2007.

[14] MACK, J.Hunter; ACEVES, Salvador M.; DIBBLE, Robert W. Demonstrating direct use of wet ethanol in a homogeneous charge compression ignition (HCCI) engine.

Energy, v. 34, n. 6, p. 782-787, 2009 
[15] SJÖBERG, Magnus; DEC, John E. Effects of EGR and its constituents on HCCI autoignition of ethanol. Proceedings of the Combustion Institute, v. 33, n. 2, p. 3031-3038, 2011.

[16] NISHI, Mina; KANEHARA, Masato; IIDA, Norimasa. Assessment for innovative combustion on HCCI engine by controlling EGR ratio and engine speed. Applied Thermal Engineering, v. 99, p. 42-60, 2016.

[17] PUTRASARI, Yanuandri; JAMSRAN, Narankhuu; LIM, Ocktaeck. An investigation on the DME HCCI autoignition under EGR and boosted operation. Fuel, v. 200, p. 447-457, 2017.

[18] MARTINS, Mario; ZHAO, Hua. 4-Stroke Multi-Cylinder Gasoline Engine with Controlled Auto-Ignition (CAI) Combustion: a comparison between Naturally Aspirated and Turbocharged Operation. SAE Technical Paper, 2008.

[19] WELLING, Orian; COLLINGS, Nick. UEGO Based Measurement of EGR Rate and Residual Gas Fraction. SAE Technical Paper, 2011.

[20] HEGARTY, Kieran et al. Fast O 2 Measurement using Modified UEGO Sensors in the Intake and Exhaust of a Diesel Engine. SAE Technical Paper, 2013.

[21] DICKINSON, Paul B. et al. Application of Fast Oxygen Sensors for Investigations into Air-Path Dynamics and EGR Distribution in a Diesel Engine. SAE Technical Paper, 2014.

[22] MARTINS, Mario et al. HCCI of Wet Ethanol on a Dedicated Cylinder of a Diesel Engine. SAE Technical Paper, 2017. 